\title{
The cognitive processing potential of infants: Exploring the impact of an early childhood development programme
}

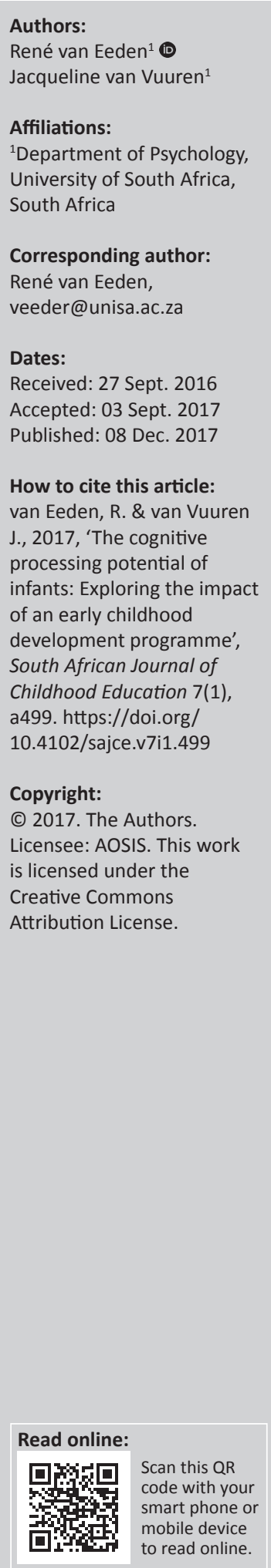

Background: Many South African learners seem unprepared for formal education, and a need for intervention during early childhood has been identified.

Aim: The present study explored the effect of infant exposure to an early childhood development programme aimed at the sensory developmental stage of the infant's brain.

Setting: Participants were recruited through local baby clinics and nursery schools in the Western Cape. Participants were from the middle-income sector and the sample consisted of 63 infants between the ages of 3 and 12 months - gender representation was approximately equal and $17 \%$ of the infants were of mixed race, $8 \%$ black and $75 \%$ white.

Methods: A pretest-posttest design was used involving an intervention group $(N=29)$ and a control group $(N=34)$ of infants. There was no known bias in group allocation. Intervention was provided in the form of the Numbers in Nappies programme and cognitive performance was assessed with the BSID (III) before and after the intervention for both groups.

Results: The intervention group showed theory expectant increases, most notably on the Cognitive Scale and the Social-Emotional Scale of the BSID (III). The performance of the intervention and the control group on the cognitive subscales (Cognitive, Language and Motor) was compared before and after the intervention. The only significant difference was on the Cognitive Scale after the intervention.

Conclusion: The findings indicate that appropriate intervention taps into the cognitive processing potential of infants, thus increasing their cognitive ability and enhancing their social-emotional functioning. The stimulation provided by parents and primary caregivers is essential in enhancing this experience-dependent development and the Numbers in Nappies programme provides a cost-effective intervention suitable for a home environment.

\section{Introduction}

Numerous psychobiological and psychosocial factors may influence development during the first years of life (Stein et al. 2015). Given that the risk factor profiles of low- and middle-income countries (LMICs) differ from those of developed countries, research on risk and protective factors in these countries is essential to ensure that children reach their full cognitive potential (MurrayKolb et al. 2014). Risk factors such as low socioeconomic status, an adverse family environment and a lack of adequate stimulation and learning opportunities for development have a cumulative negative impact on development (Grantham-McGregor et al. 2007; Murray-Kolb et al. 2014; Rademeyer \& Jacklin 2013; Saloojee \& Pettifor 2005; Stein et al. 2015). These and other negative social influences characterise many communities in South Africa. Early intervention is therefore essential to counter the effect of these factors and prevent or address developmental delays.

Interventions to help children at emotional, medical and nutritional level have been initiated in some local communities. From an intellectual support perspective, the government provides education from Reception to Grade 12, and approximately a third of younger age groups also attend an educational institution (Statistics South Africa 2014). However, in socioeconomically disadvantaged settings, barriers to learning are already present in the early grades (Wildschut, Moodley \& Aronstam 2016). Many South African learners seem unprepared for formal education and there is a need for development programmes that are implemented in early childhood. Funding limitations, lack of public facilities for early childhood activities and limited community participation have been listed as factors that prevent a standardised system for supporting such programmes (Department of Basic Education 2001). Privately initiated educational programmes 
are available to preschool and school-aged children but there is limited availability of programmes for infants and toddlers that are accessible in ways other than through educational institutions.

Early mental development has been linked to preschool cognitive and behavioural outcomes (Hsiao \& Richter 2014), and the intellectual stimulation of infants and young children by means of early childhood development programmes has been shown to correlate with achievement in school and in life (Frede et al. 2009). Furthermore, early interventions that focus on the development of skills in infants and young children could increase the effectiveness of later interventions (Heckman 2006). Richter, Mabaso and Hsiao (2016) referred to the greater prevalence of developmental delays in LMICs and the consequent need for early identification and prevention of delays.

Development in the brain begins within the first month of conception, and by 6 months of the gestational age, most neurons of the mature brain exist (Huttenlocher \& Dabholkar 1997). Synapses are formed across neurons and dendrites to enable the storage of new information as the brain is stimulated by experiences from the environment, and a process of pruning, in turn, eliminates unnecessary and superfluous or surplus (i.e. inactive) connections. Synaptogenesis begins early in gestation but exuberant synaptogenesis characterises the period before birth until approximately 2 years of age. The first year of life is critical for brain growth as this is a time when many of the sensitive periods for the connection of the neural pathways overlap. Development of the visual and auditory cortex, as well as the receptive language and speech production areas of brain development, peaks during this period (GranthamMcGregor et al. 2007). In the first year, there is also rapid development in terms of infant motor coordination and balance (Knickmeyer et al. 2008). Theoretically, activity and sensorimotor experience are necessary for cognitive development, and research supports the dependent development of motor and cognitive skills (e.g. Campos et al. 2012). The aim of the intervention programme used in the present study was to stimulate the infant at the stage where synapses are being connected through auditory and visual stimulation by strengthening synapse connections for numbers, shapes and colours.

In the Numbers in Nappies programme flashcards of numbers, shapes and colours are used to stimulate the image brain (Van Vuuren 2014). The latter is dominant between the ages of 0 and 3 (Shichida 1993) and allows immediate access to information that was stored in the memory (i.e. what the infant saw on the flashcard). The left brain relies on repetition to absorb information and the concepts of numbers, shapes and colours are therefore taught in isolation and repetitively. The number concepts are presented visually by means of the flashcards and auditorily through the parent's voice, thus using these dominant means to strengthen synaptic connections during the first year of life.
During this period, vulnerability to negative influences is high, but there are also great opportunities for unlocking potential with the assistance of intervention programmes (Allen \& Duncan Smith 2008). The impact of the social environment and the caregiver relationship on cognitive and social-emotional development is supported by theory and practice (e.g. Murray et al. 2016). Vygotsky (1978) distinguished between lower mental functions present at birth and complex mental activities that require mediation to develop. Developmental opportunities are created in the way the caregiver communicates and stimulates the child. In the early days of an infant's life, he or she can recognise the mother's voice and prefers it over other sounds (Cooper \& Aslin 1994). Studies with infants and toddlers show that reduced verbal interaction could cause delays in language development (Christakis et al. 2009), whereas regular reading to young children improves their language ability (Richert et al. 2010). Given the value of personal interaction in early stimulation and infant learning, this was used as the medium for presenting the present programme.

The Numbers in Nappies programme comprises individual experiences provided by the parents as opposed to typical everyday experiences that contribute to brain development. A review of existing programmes showed that there are locally developed or adapted programmes available but these are primarily aimed at infants with a specific delay (e.g. Russel et al. 2016). These programmes therefore target developmental functions associated with this delay. It was decided rather to develop a programme comprising a set of theoretically based activities aimed at the broader infant population.

The present study explored the effect of infant exposure to an early childhood development programme aimed at the sensory developmental stage of the infant's brain. The Numbers in Nappies programme consists of flashcards with numbers, shapes and colours and the programme stimulates development through parent involvement. The objective of this study was to explore whether early infant exposure to brain stimulation in this form increases the infant's cognitive processing potential.

\section{Research method and design Study design}

An intervention group participated in the Number in Nappies programme and their performance on the composite scales of the Bayley Scales of Infant and Toddler Development - Third Edition (BSID - III) was compared before and after the intervention. The performance of the intervention group was also compared to that of a control group before the intervention (to ensure that the groups were matched in terms of ability) and after the intervention (to determine the impact of the programme).

\section{Study population and sampling strategy}

Purposive sampling was used to select infants between the ages of 3 and 12 months at the commencement of the research. 
Only infants who had not been diagnosed with any developmental problems and who had no history of any health problems or physical defects were included in the sample. Potential participants were recruited through local baby clinics and nursery schools in the Western Cape. Interested parents were interviewed and the details of the study were provided.

The content and presentation format of the Numbers in Nappies programme make it intellectually and economically accessible to infants across all socioeconomic sectors. Participants from different income groups partook in a pilot study to refine the presentation procedures. However, to limit differences in developmental opportunities, it was decided to focus on the middle-income sector during this initial evaluation of the impact of the programme on the cognitive processing potential of infants. The definition of Statistics South Africa (2002-2009) was used to screen participants in terms of whether they lived in formal housing and whether this housing included electricity, running water, etc.

The realised sample consisted of 63 infants $(17 \%$ mixed race, $8 \%$ black and $75 \%$ white), with 29 infants in the intervention group and 34 infants in the control group. Gender representation was approximately equal and the two groups were matched as far as possible in terms of age, gender and race (see Van Vuuren 2014). Practical considerations did in some cases influence group membership of a parent and infant, but this was not expected to bias the outcome and allocation to the intervention group and the control group was regarded as random.

\section{Intervention programme}

The Numbers in Nappies programme was based on concepts of numeracy in infants and young children, as explained by Doman and Doman (2005) and Shichida (1993). The right brain or image brain is dominant between the ages of 0 and 3 . Photographic memory is used to recall information, thus allowing immediate access to information stored in the memory. The more logical left brain relies on repetition to absorb information. The methodology used by Doman and Doman and Shichida involves showing simple flashcards with red dots to teach basic mathematics principles (i.e. quantity recognition and equations) using quantity, imaging and problem solving. The right brain enables recall of information from the flashcards, whereas the left brain is involved through consistent daily flashcard exposure over 2-3 months. The Numbers in Nappies programme was specifically developed as part of the present study and it has therefore not been previously evaluated.

The Numbers in Nappies programme exposes the infant to numbers, shapes and colours at a stage when learning takes place through visual, auditory and sensory stimulation. Imitation, problem solving, memory, number sense, and classification and attention maintenance are emphasised (Halberda, Mazzocco \& Feigenson 2008). These areas of cognitive development can be seen as the foundation for understanding numerical concepts and are important in the introduction of numbers, shapes and colours. Ten flashcards of shapes, colours or numbers are shown to the infant for approximately 2-3 s for each flashcard. The colours red, blue, green, yellow, orange, pink, purple, black, brown and grey are included. The shapes comprise the triangle, square, circle, diamond, rectangle, oval, semi-circle, star, hexagon and cross. In addition to showing dots representing the numbers $1-30$, the number flashcards are also used to introduce the concepts of addition, subtraction, multiplication, greater than and less than. Stimuli are presented visually by means of the flashcard method and auditorily, through the parent's voice. The intervention programme is applied on a daily basis, two to three times a day, for approximately 1 min per session, over an average of 60 days. There is variation in the flashcards shown over this period.

The programme promotes bonding between the parent and the baby. The programme avoids the use of technology and the stimulation is presented through direct communication in a positive learning environment. Emphasis is placed on the infant's comfort and the need for affection and encouragement from the parent.

\section{Measuring instrument}

The instrument known as the Bayley Scales of Infant and Toddler Development was used in the present study as it enables a comprehensive assessment of the relevant cognitive, language and motor developmental areas. The third edition of the test, the BSID (III), was released in 2006, and the U.S. normative sample comprised typically developing children between 1 and 42 months of age (Bayley 2006). Information supporting the reliability and validity of the test was reported in the technical manual (Bayley 2006). Confirmation of the construct validity was provided by means of factor analysis and correlations with tests measuring related constructs (Albers \& Grieve 2007). Special group studies included research on prematurity, small for gestational age, Down syndrome, pervasive developmental disorder, asphyxia, cerebral palsy and language impairment (Bedford, Walton \& Ahn 2013).

Research in South Africa and other African countries has supported the value of the Bayley Scales in the local context (e.g. Brown 2009; Hutchings \& Potterton 2014; Rademeyer \& Jacklin 2013; Richter et al. 2016). Moderate support was found for the predictive validity of the Bayley Scales in a longitudinal study involving primarily black African infants residing in an urban area in South Africa (Richter et al. 2016). A sample with similar demographics was studied by Rademeyer and Jacklin (2013) but they specifically used the BSID (III) that was also used in the present study. Their findings also provided support for the use of the test in the South African context.

The Bayley Scales present the infant with situations that produce observable responses. The rationale is that even if a child cannot speak, such responses give an indication of how the child thinks, feels and interacts with the world 
around him or her. Albers and Grieve (2007) referred to a number of concepts of early cognition that were considered during the development of the BSID (III). These included concepts traditionally associated with the Bayley Scales, such as play, information processing and number concepts. In addition, more recent research on information processing and preverbal intelligence was also consulted.

The BSID (III) consists of the Cognitive Composite Scale (91 items), the Language Composite Scale (97 items) and the Motor Composite Scale (138 items). The Language Composite Scale comprises the receptive communication subtest (49 items) and the expressive language subtest (48 items), while the Motor Composite Scale comprises the fine motor subtest (66 items) and the gross motor subtest (72 items). The Cognitive Composite Scale assesses, amongst others, sensorimotor development, exploration and manipulation, object relatedness, concept formation, memory, habituation, visual acuity, visual preference and object permanence. From a professional point of view, the addition of a separate language scale can be regarded as an improvement (Albers \& Grieve 2007). In the Language Composite Scale, a distinction is made between the ability to understand and respond to verbal stimuli and the ability to vocalise, communicate and name pictures and objects. The Motor Composite Scale distinguishes between fine and gross motor skills. The former includes skills involved in prehension, perceptual-motor integration, motor planning and motor speed while static positioning, dynamic movement and the quality of movement are assessed, amongst others, in the case of the latter.

In addition to the three cognitive subscales (the focus of the present study), each infant's parent or primary caregiver is required to complete two extra scales, the Social-Emotional Scale and the Adaptive Behaviour Scale, as well as a Behaviour Observation Inventory. The assessor also completes this inventory.

Subtest scores are converted to scaled scores with a mean of 10 and standard deviation of 3 , while the scores for the composite scales are converted to composite scores with a mean of 100 and a standard deviation of 15 .

The dynamic nature of child development implies challenges for the effective assessment thereof (Bedford et al. 2013). The complexity of each area of development needs to be considered together with the interdependence of the different domains (Meisels \& Atkins-Burnett 1999). Development could furthermore be affected by numerous factors and there is not necessarily continuity in development. 'Children may develop at different rates at different times' (Grieve \& Foxcroft 2013:271). This situation impacts negatively on the predictive validity of infant tests. However, this was not deemed a problem in the present study given the short time period between pre- and post-testing. Including a control group furthermore enabled the researchers to limit the impact of factors other than the intervention programme.

\section{Data collection}

An occupational therapist assessed all the infants with the BSID (III). A clinical psychologist supervised the assessment process and the participating parents as well as the researcher were also present during the assessment. The infants were assessed in their home language where possible, and each assessment took approximately an hour. The parents of the infants in the intervention group subsequently underwent training in the Numbers in Nappies programme and the programme was implemented. After approximately 2 months, the BSID (III) was again administered to all the infants. At this stage, the control group was also afforded an opportunity to participate in the Numbers in Nappies programme on a voluntary basis. Commitment was ensured by providing the parents with detail information on the study before it commenced. During the implementation phase, nine mothers from the intervention group also participated in discussions that were held every second week. The aim was to monitor parent participation in the programme and also to receive feedback on their experience of the programme and on their infants' responses and development.

\section{Data analysis}

To determine the effect of the intervention programme (the independent variable), the cognitive performance (dependent variable) of the infants in the intervention group was compared before and after the intervention programme was implemented. The performance of the infants in the intervention group was also compared to that of the control group before implementation (to set a baseline) and after implementation of the programme (to determine the effect thereof).

Descriptive statistics were calculated for all five composite scales (Cognitive, Language, Motor, Adaptive Behaviour and Social-Emotional) of the BSID (III). Within-group and between-group comparisons were done for the cognitive subscales using non-parametric tests (because the sample size was less than 100 participants). The Wilcoxon signed-rank test and the Mann-Whitney U test were used, respectively.

Preceding the comparisons on the cognitive scales, the effects of age and gender were explored to exclude possible alternative explanations as far as possible. No significant differences were found for the intervention or the control group. However, trends in the data indicated that the combined effect of age and gender had to be further explored. A larger sample would be preferable for such analysis.

\section{Ethical considerations}

Ethical clearance was obtained from the supervising university. Participation was voluntary and a consent form was signed by the participating parents. This included references to confidentiality, non-maleficence and beneficence. Participants were also free to withdraw at any stage during the study. The safety and comfort of the infants 
and the parents were a priority during the assessment and the intervention. The study focused on the group as a whole, but if there were any concerns regarding an individual infant, he or she was referred for further assessment.

\section{Results}

As indicated in Table 1, the performance on all subscales was similar for the two groups at the first assessment. Scores on the cognitive subscales (Cognitive, Language and Motor) and the behavioural subscale (Adaptive Behaviour) were average, with high scores for the Social-Emotional Scale. Scores ranged between 96.38 and 119.38 for the intervention group and between 94.91 and 115.91 for the control group. There were numerical increases on all subscales for the intervention group after the intervention with scores ranging between 99.27 and 130.63 (Table 1). This was especially clear in the case of the Cognitive Scale and the Social-Emotional Scale. In the case of the latter, performance was now in the very superior range.

The Wilcoxon signed-rank matched pairs test was used to further explore the differences between the scores on the cognitive subscales (the focus of the present study) for the intervention group before and after the intervention. The composite scores for the Cognitive Scale were rank ordered and ranks (negative ranks, positive ranks and ties) were compared. The results in Table 2 show that there was a significant increase after the intervention $(Z=-4.32, p<0.01)$. However, the intervention programme did not have a statistically significant effect in the case of the Language Scale $(Z=-1.68, p=0.09)$ or the Motor Scale $(Z=-0.76, p=0.45)$.

The Mann-Whitney $U$ test was used to compare the performance on the cognitive subscales of the intervention group to that of the control group both before and after the intervention. As seen in Table 3, no significant differences

TABLE 1: Means and standard deviations of the composite scores for the intervention group and the control group for each subscale.

\begin{tabular}{|c|c|c|c|c|c|c|}
\hline \multirow[t]{2}{*}{ Subscale } & \multirow[t]{2}{*}{ Group } & \multirow[t]{2}{*}{$N$} & \multicolumn{2}{|c|}{ First assessment } & \multicolumn{2}{|c|}{ Second assessment } \\
\hline & & & $M$ & SD & $M$ & SD \\
\hline \multirow[t]{2}{*}{ Cognitive } & Intervention & 29 & 97.97 & 9.34 & 109.90 & 12.92 \\
\hline & Control & 34 & 98.21 & 7.76 & 99.56 & 8.11 \\
\hline \multirow[t]{2}{*}{ Language } & Intervention & 29 & 103.35 & 12.14 & 107.03 & 10.91 \\
\hline & Control & 34 & 103.18 & 11.43 & 102.23 & 13.70 \\
\hline \multirow[t]{2}{*}{ Motor } & Intervention & 29 & 102.07 & 8.98 & 104.52 & 14.10 \\
\hline & Control & 34 & 99.12 & 12.20 & 100.91 & 13.64 \\
\hline \multirow[t]{2}{*}{ Adaptive behaviour } & Intervention & 29 & 96.38 & 9.18 & 99.27 & 9.24 \\
\hline & Control & 34 & 94.91 & 9.77 & 97.13 & 9.77 \\
\hline \multirow[t]{2}{*}{ Social-emotional } & Intervention & 29 & 119.38 & 6.82 & 130.63 & 12.36 \\
\hline & Control & 34 & 115.91 & 12.73 & 119.38 & 6.82 \\
\hline
\end{tabular}

TABLE 2: Statistics ${ }^{\mathrm{a}, \mathrm{b}}$ for subscale comparisons in the intervention group $(N=29)$ before and after the intervention programme.

\begin{tabular}{lccc}
\hline & Cognitive2 Cognitive1 & Language2 Language1 & Motor2 Motor1 \\
\hline$Z$ & $-4.32^{c}$ & $-1.68^{c}$ & $-0.76^{c}$ \\
$p$ (two-tailed) & $0.00^{* *}$ & 0.09 & 0.45 \\
\hline
\end{tabular}

${ }^{a}$ Control/intervention = intervention.

${ }^{b}$ Wilcoxon signed-rank test.

'Based on negative ranks.

$* * p \leq 0.05$. were found before the intervention on any of the subscales (Cognitive Scale $U=482, Z=-0.16 ; p=0.83$; Language Scale $U=507, Z=0.19, p=0.85$; Motor Scale $U=582, Z=1.23$, $p=0.22)$. However, Table 4 shows that the intervention group scored significantly higher after the intervention on the Cognitive Scale $(U=732, Z=3.32, p<0.01)$. No significant effect of the intervention programme was found in the case of the Language Scale $(U=622, Z=1.78, p=0.07)$ or the Motor Scale $(U=595, Z=1.14, p=0.16)$.

\section{Discussion}

The objective of the study was to explore the effect of early infant exposure to the Numbers in Nappies programme on the infants' cognitive performance as measured on the BSID (III). An intervention and a control group of infants were assessed with the BSID (III) at the beginning of the study and again after approximately two months. In this time period, the Numbers in Nappies programme was implemented for the intervention group.

There were numerical increases on the cognitive subscales for the intervention group after the implementation of the programme. However, this increase was only significant in the case of the Cognitive Scale. When comparing the performance of the two groups on the cognitive subscales, no significant differences were found before the intervention. Only in the case of the Cognitive Scale was a significant difference found after the intervention. No significant result was found for the Language Scale and the Motor Scale. Numerical increases were noted for the intervention group on the Adaptive Behaviour Scale and the Social-Emotional Scale.

The significant improvement on the Cognitive Scale for those infants who did the intervention programme is supported by various studies (e.g. Campbell \& Ramey 1994; Chickgoudar \& Khadi 2001; Murray et al. 2016). In these studies, significant improvements were reported in the cognitive development of the infants in the experimental group after intervention. Greater awareness of the role of cognitive stimulation was probably created by the intervention programme, resulting in the significantly higher score for the intervention group in comparison with the control group.

TABLE 3: Test statistics for the comparison of the intervention $(N=29)$ and control groups $(N=34)$ for each subscale before the intervention.

\begin{tabular}{lccc}
\hline & Cognitive & Language & Motor \\
\hline$N$ & 63 & 63 & 63 \\
Mann-Whitney U & 482 & 507 & 582 \\
$Z$ & -0.16 & 0.19 & 1.23 \\
$p$ (two-tailed) & 0.83 & 0.85 & 0.22 \\
\hline
\end{tabular}

TABLE 4: Test statistics for the comparison of the intervention $(N=29)$ and control groups $(N=34)$ for each subscale after the intervention.

\begin{tabular}{lccc}
\hline & Cognitive & Language & Motor \\
\hline$N$ & 63 & 63 & 63 \\
Mann-Whitney U & 732 & 622 & 595 \\
$Z$ & 3.32 & 1.78 & 1.14 \\
$p$ (two-tailed) & $0.00^{* *}$ & 0.07 & 0.16 \\
\hline
\end{tabular}

** $p \leq 0.05$ 
The numerical increases in language and motor composite scores in the case of the intervention group reflected the expected trend after the infants' exposure to the stimulation programme. These results also provided support for the link that has been reported between cognitive and motor development (Campos et al. 2012). There were, however, no significant differences when comparing performance on these subscales before and after the intervention. It is possible that caregivers encourage language and motor skills more than cognitive abilities in the early months of life (Rademeyer \& Jacklin 2013). Natural encouragement in these two areas could partly explain a less pronounced impact when additional stimulation is provided. The type of stimulation and the nature of the sample could also have played a role. Chickgoudar and Khadi (2001) found significant improvements in motor development when using three-dimensional activities as stimulation. Based on the findings by Russel et al. (2016), it is also hypothesised that the effect of the intervention programme would be more pronounced in the case of diagnosed developmental delays.

The emphasis of the present study was on cognitive performance, but it was interesting to note that although there were no significant increases in the case of adaptive behaviour, a significant increase was evident in terms of the infants' social-emotional functioning after exposure to the intervention programme. Adaptive behaviour is usually assessed in circumstances where there are developmental concerns, because an infant's adaptive behaviour is linked to the other areas of development (Windsor et al. 2007). A more noticeable intervention effect would be expected under these circumstances. The absence of developmental delays in the present study implied that differences in the composite score means for the two groups on the behavioural scale were not necessarily anticipated. A slight numerical increase in the second assessment for both groups could be because of the fact that the infants were slightly older.

The increase in social-emotional functioning from 'high' to 'very superior' could be attributed to the added stimulation provided by the parents during the intervention. The mediating effect of carer-infant interactions in the case of both cognitive and social-emotional outcomes has been reported previously (e.g. Murray et al. 2016).

Gender and age did not seem to affect the performance of the present sample. However, this finding should be further explored with a larger sample of infants. It would be important to consider a potential interactive effect between these two variables (Rademeyer \& Jacklin 2013).

This study should be regarded as a foundation for further research, and the results should not be generalised without replication on a larger and more representative sample in terms of ethnicity, socioeconomic status and regional area. Justification was provided for including participants from the middle-income sector, but it is especially the risk factors in less privileged communities that necessitate this type of intervention. The role of the test administrator is more pronounced in infant assessment, and repetition in different contexts is therefore advisable. A more structured evaluation of the implementation of the programme by the parents is also recommended.

Infant tests of cognitive ability per se are not regarded as the most effective predictive index of future abilities and functioning, because of the strong impact of the child's home environment (Meisels \& Atkins-Burnett 1999). On the other hand, supportive results have been found for the long-term effect of early development programmes (Frede et al. 2009; Hsiao \& Richter 2014) probably because these programmes target the contextual impact. Longitudinal studies are nevertheless recommended to determine if the foundations laid in the first year of life with the Numbers in Nappies programme have an impact later on in the child's school career.

\section{Conclusions}

The cognitive development of infants can be improved when they are nurtured in an environment that is physically and socially encouraging. Socioeconomic constraints imply that many South African parents struggle to provide cognitively stimulating homes for their children (Brown 2009), and without early intervention, many children born into economically disadvantaged families fail to reach their potential (Ramey \& Ramey 1999). A cost-effective intervention programme could enable parents to provide educational stimulation for their infants in a home environment. Intervention at such an early age does not form part of the formal education system, and support from private and nongovernment organisations to parents and primary caregivers would imply a potentially broader implementation of the Numbers in Nappies programme.

The physical and social environments play a role in the acquisition of cognitive, language, motor and social skills, but infants are mainly interested in learning from people. The individual experiences provided in the present programme create opportunities for new growth and refine existing structures, thus enhancing experience-dependent development. The findings of this study support the view that appropriate intervention taps into the cognitive processing potential of infants, therefore increasing their cognitive ability and enhancing their social-emotional functioning.

\section{Acknowledgements}

The authors want to thank Hendrik Marais and Karina Strydom, founders of Brain Boosters, for their financial assistance with the study. Their passion and energy in helping improve pre-school education in South Africa is truly inspirational.

\section{Competing interests}

The authors declare that they have no financial or personal relationships that may have inappropriately influenced them in writing this article. 


\section{Authors' contributions}

J.v.V. conducted the research for her master's degree. R.v.E. was the supervisor for this study and she also consulted additional literature and conducted confirmatory analyses in preparation for the article. Both authors contributed to the writing of the article.

\section{References}

Albers, C.A. \& Grieve, A.J., 2007, 'Test reviews: Bayley Scales of Infant and Toddler Development - Third Edition', Journal of Psychoeducational Assessment 25(2) 180-198. https://doi.org/10.1177/0734282906297199

Allen, G. \& Duncan Smith, I., 2008, Early intervention: Good parents, great kids, better citizens, Centre for Social Justice and the Smith Institute, London.

Bayley, N., 2006, Bayley Scales of Infant and Toddler Development - Third Edition: Technical manual, Harcourt Assessment, San Antonio, TX.

Bedford, H., Walton, S. \& Ahn, J., 2013, Measures of child development: A review, University College London Institute of Child Health, London, UK.

Brown, D., 2009, 'Developmental differences between pre-term and full-term 18 month olds', MSc dissertation, Department of Physiotherapy, University of the Witwatersrand.

Campbell, F. \& Ramey, G.T., 1994, 'Effects of early intervention and intellectual and academic achievement: A follow-up study of children from low-income families', Child Development 65(2), 684-698. https://doi.org/10.2307/1131410

Campos, D.P.T., Gonçalves, V.M.G., Guerreiro, M.M., Santos, D.C., Goto, M.M.F., Arias, A.V. et al., 2012, 'Comparison of motor and cognitive performance in infants during the first year of life', Pediatric Physical Therapy 24(2), 193-197. https://doi. org/10.1097/PEP.0b013e31824d2db7

Chickgoudar, P.S. \& Khadi, P.B., 2001, 'Impact of intervention on motor and mental development of rural infants (9-15 months)', Karnataka Journal of Agricultural development of rural in
Sciences 14(2), 549-552.

Christakis, D.A., Gilkerson, J., Richards, J.A., Zimmerman, F.J., Garrison, M.M., Xu, D. et al., 2009, 'Audible television and decreased adult words, infant vocalizations, and
conversational turns: A population-based study', Archives of Pediatrics and Adolescent Medicine 163, 554-558. https://doi.org/10.1001/archpediatrics. 2009.61

Cooper, R.P. \& Aslin, R.N., 1994, 'Developmental differences in infant attention to the spectral properties of infant-directed speech', Child Development 65(6), 16631667. https://doi.org/10.2307/1131286

Doman, G. \& Doman, J., 2005, How to teach your baby math: The gentle revolution, Square One, New York.

Department of Basic Education, 2001, Education white paper 5 on early childhood education: Meeting the challenge of early childhood development in South Africa, Department of Basic Education, Pretoria, viewed 06 September 2016, from http:///www.education.gov.za/Resources/Legislation/WhitePapers.aspx

Frede, E., Jung, K., Barnett, W.S. \& Figueras, A., 2009, The APPLES blossom: Abbott preschool program longitudinal effects study (APPLES) preliminary results through 2nd grade (Interim Report), National Institute for Early Education Research Rutgers University, New Brunswick, NJ, viewed 20 September 2012, from http:// ieer.org/pdf/apples_second_grade_results.pdf

Grantham-McGregor, S., Cheung, Y.B., Cueto, S., Glewwe, P., Richter, L., Strupp, B. \& The International Child Development Steering Group, 2007, 'Developmental potential in the first 5 years for children in developing countries', The Lancet 369(9555), 60-70. https://doi.org/10.1016/S0140-6736(07)60032-4

Grieve, K.W. \& Foxcroft, C., 2013, 'Factors affecting assessment results', in C. Foxcroft \& G. Roodt (eds.), Introduction to psychological assessment in the South African context (4th ed.), pp. 269-285, Oxford University Press, Cape Town.

Halberda, J., Mazzocco, M.M. \& Feigenson, L., 2008, 'Individual differences in nonverbal number acuity correlate with maths achievement', Nature 455, 665-668. https://doi.org/10.1038/nature07246

Heckman, J.J., 2006, 'Skill formation and the economics of investing in disadvantaged children', Science 312, 1900-1902. https://doi.org/10.1126/science.1128898

Hsiao, C. \& Richter, L.M., 2014, 'Early mental development as a predictor of preschool cognitive and behavioural development in South Africa: The moderating role of maternal education in the Birth to Twenty Cohort', Infants \& Young Children 27(1) 74-87. https://doi.org/10.1097//YC.0000000000000002
Hutchings, J. \& Potterton, J., 2014, 'Development delay in HIV exposed infants in Harare, Zimbabwe', Vulnerable Children and Youth Studies 9(1), 43-55. https:// doi.org/10.1080/17450128.2013.778440

Huttenlocher, P.R. \& Dabholkar, A.S., 1997, 'Regional differences in synaptogenesis in human cerebral cortex', Journal of Comparative Neurology 387(2), 167-178. https://.doi.org/10.1002/(SICI)1096-9861(19971020)387:2<167::AIDhttps://.doi.org/1

Knickmeyer, R.C., Gouttard, S., Kang, C., Evans, D., Wilber, K., Smith, J.K. et al., 2008 'A structural MRI study of human brain development from birth to 2 years', The Journal of Neuroscience 28(47), 12176-12182. https://doi.org/10.1523/ The Journal of Neuroscienc
JNEUROSCI.3479-08.2008

Meisels, S.J. \& Atkins-Burnett, S., 1999, 'Assessing intellectual and affective development before age three: A perspective on changing practices', Food and Nutrition Bulletin 20(1), 23-33.

Murray, L., De Pascalis, L., Tomlinson, M., Vally, Z., Dadomo, H., MacLachlan, B. et al., 2016, 'Ramdomized controlled trial of a book-sharing intervention in a deprived South African community: Effects on carer-infant interactions, and their relation to infant cognitive and socioemotional outcome', Journal of Child Psychology and Psychiatry 57(12), 1370-1379. https://doi.org/10.1111/jcpp.12605

Murray-Kolb, L.E., Rasmussen, Z.A., Scharf, R.J., Rasheed, M.A., Svensen, E., Seidman, J.C. et al., 2014, 'The MAL-ED cohort study: Methods and lessons learned when assessing early child development and caregiving mediators in infants and young children in 8 low- and middle-income countries', Clinical Infectious Diseases 59(Suppl 4), S261-S272. https://doi.org/10.1093/cid/ciu437

Rademeyer, V. \& Jacklin, L., 2013, 'A study to evaluate the performance of black South African urban infants on the Bayley Scales of Infant Development III', South African Journal of Child Health 7(2), 54-59. https://doi.org/10.7196/SAJCH.547

Ramey, C.T. \& Ramey, S.L., 1999, Right from birth: Building your child's foundation for life, Goddard Press, New York.

Richert, R.A., Robb, M.B., Fender, J.G. \& Wartella, E., 2010, 'Word learning from baby videos', Archives of Pediatric and Adolescent Medicine 164(5), 432-437. https:// doi.org/10.1001/archpediatrics.2010.24

Richter, L., Mabaso, M. \& Hsiao, C., 2016, 'Predictive power of psychometric assessments to identify young learners in need of early intervention: Data from the Birth to Twenty Plus Cohort, South Africa', South African Journal of Psychology the Birth to Twenty Plus Cohort, South Africa', South African Journ
46(2), 175-190. https://doi.org/10.1177/0081246315599476

Russel, D.C., Van Heerden, R., Van Vuuren, S., Venter, A. \& Joubert, G., 2016, 'The impact of the "Developmental Resource Stimulation Programme" (DRSP) on children with Down syndrome', South African Journal of Occupational Therapy children with Down syndrome', South African Journal of Occupation
46(1), 33-40. https://doi.org/10.17159/2310-3833/2016/v46n1a8

Saloojee, H. \& Pettifor, J.M., 2005, 'International child health: 10 years of democracy in South Africa; the challenges facing children today', Current Paediatrics 15, 429436. https://doi.org/10.1016/j.cupe.2005.06.012

Shichida, M., 1993, Brain education in infancy: Theory and practice, Shichida Child Education, Japan.

Statistics South Africa, 2002-2009, General Household Survey, Statistics South Africa, Pretoria, viewed April 2012, from http://www.statssa.gov.za/publications/P0302/ P03022011.pdf

Statistics South Africa, 2014, General Household Survey, Statistics South Africa, Pretoria, viewed 05 September 2016, from http://www.statssa.gov.za/ publications/P0318/P03182014.pdf

Stein, D.J., Koen, N., Donald, K.A., Adnams, C.M., Koopowitz, S., Lund, C. et al., 2015 Investigating the psychosocial determinants of child health in Africa: The Drakenstein Child Health Study', Journal of Neuroscience Methods 252, 27-35. https://doi.org/10.1016/j.jneumeth.2015.03.016

Van Vuuren, J., 2014, 'The cognitive processing potential of infants: A study of the effect of early infant exposure to numbers, shapes and colours', MA dissertation, Department of Psychology, University of South Africa.

Vygotsky, L.S., 1978, Mind and society: The development of higher mental processes, Harvard University Press, Cambridge, MA.

Wildschut, Z., Moodley, T. \& Aronstam, S., 2016, 'The baseline assessment of Grade 1 learners' literacy skills in a socio-economically disadvantaged school setting' South African Journal of Childhood Education 6(1), a340. https://doi.org/10.4102/ sajce.v6i1.340

Windsor, J., Glaze, L.E., Koga, S.F. \& The BEIP Core Group, 2007, 'Language acquisition with limited input: Romanian institution and foster care', Journal of Speech Language, and Hearing Research 50, 1365-1381. https://doi.org/10.1044/1092 4388(2007/095) 